\title{
Cost-Utility Analysis of Ravulizumab Compared with Eculizumab in Adult Patients with Paroxysmal Nocturnal Hemoglobinuria
}

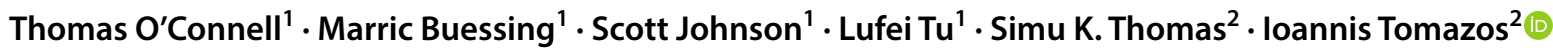

Published online: 10 June 2020

(c) The Author(s) 2020

\begin{abstract}
Background Paroxysmal nocturnal hemoglobinuria, characterized by intravascular hemolysis and venous thrombosis, can be managed with eculizumab, an inhibitor of the complement system; however, patients may periodically experience breakthrough hemolysis. Ravulizumab is a newly approved treatment for paroxysmal nocturnal hemoglobinuria that may reduce breakthrough hemolysis risk, thus improving health-related quality of life and reducing treatment costs.

Objective The objective of this study was to compare the costs and benefit of treatment with ravulizumab vs eculizumab in adult patients with paroxysmal nocturnal hemoglobinuria, from a US payer perspective.

Methods A cost-utility analysis was conducted using a semi-Markov model, informed by clinical experts. Lifetime costs and benefit (quality-adjusted life-years) (both discounted at 3\% per annum) and incremental cost-effectiveness ratios were estimated, over a lifetime horizon. Results are reported for an entire treated population and subgroups of eculizumab treatment history. Scenario analyses were characterized by assumptions of non-inferiority between treatments, in terms of breakthrough hemolysis incidence and blood transfusion requirements, and of variations in eculizumab dosing adjustments used in response to breakthrough hemolysis.

Results In the base-case analysis for the overall population, there was a positive impact on health-related quality of life (quality-adjusted life-year gain of 1.67) and costs were lower $(-\$ 1,673,465)$, for ravulizumab vs eculizumab. This led to a negative incremental cost-effectiveness ratio $(-\$ 1,000,818$, indicating cost savings per quality-adjusted life-year gained). Health-related quality-of-life improvement and cost savings were also observed in all cohorts and scenario analyses.

Conclusions In adults with paroxysmal nocturnal hemoglobinuria, ravulizumab is associated with improved health-related quality of life and provides a large cost saving from the perspective of a US payer, when compared with eculizumab.
\end{abstract}

\section{Introduction}

Paroxysmal nocturnal hemoglobinuria ( $\mathrm{PNH})$ is a rare, progressively debilitating, life-threatening disease characterized by intravascular hemolysis and venous thrombosis. Patients may present with varying symptoms, including significant fatigue or weakness, shortness of breath, hemoglobinuria, abdominal pain, bone marrow suppression, thrombosis, and renal insufficiency $[1,2]$.

Electronic supplementary material The online version of this article (https://doi.org/10.1007/s40273-020-00929-z) contains supplementary material, which is available to authorized users.

\footnotetext{
Ioannis Tomazos

Ioannis.Tomazos@alexion.com

1 Medicus Economics, Boston, MA, USA

2 Alexion Pharmaceuticals, Boston, MA, USA
}

Eculizumab (Soliris ${ }^{\circledR}$; Alexion Pharmaceuticals, Inc., New Haven, CT, USA), a humanized monoclonal antibody administered via intravenous infusion, is the first complement inhibitor and regulatory-approved treatment for PNH [3], and has been shown to reduce intravascular hemolysis and its clinical complications [4]. Long-term experience with eculizumab has demonstrated that it is efficacious and well tolerated [5]; despite this, $5-27 \%$ of patients receiving the approved dosage of eculizumab during long-term treatment may experience breakthrough hemolysis (BTH) events [5-8].

Breakthrough hemolysis is characterized by the return of intravascular hemolysis and the reappearance of classical PNH symptoms [2]. Such events may be categorized as: (1) BTH as a result of suboptimal free C5 inhibition, indicated by elevated plasma free $\mathrm{C} 5$ ( $\geq 0.5 \mu \mathrm{g} / \mathrm{mL}$, according to the threshold associated with complete inhibition of hemolysis in vitro); (2) BTH related to a complement-amplifying 


\section{Key Points for Decision Makers}

Patients with paroxysmal nocturnal hemoglobinuria treated with ravulizumab experience a health benefit when compared with those treated with eculizumab. This benefit results from a reduced treatment burden (i.e., a less frequent intravenous administration regimen), fewer breakthrough hemolysis events, and reduced blood transfusion requirements.

In the treatment of paroxysmal nocturnal hemoglobinuria, ravulizumab costs substantially less than eculizumab from a US payer perspective. Drivers of cost savings include: (1) ravulizumab has significantly lower average, labeled maintenance-year costs (10\% savings compared with eculizumab at list price) and (2) patients treated with eculizumab may require higher-than-labeled eculizumab dosing over time for management of breakthrough hemolysis events.

condition (CAC), defined as BTH due to an inciting event (e.g., infection, trauma, or surgery); or (3) BTH unrelated to elevated $\mathrm{C} 5$ and without a reported time-matched CAC. In some patients treated with eculizumab who experience a BTH event due to suboptimal free C5 inhibition, BTH has been managed by adjusting the dosage or frequency of maintenance doses ('up-dosing') of eculizumab to achieve and maintain efficacy of treatment [9].

Ravulizumab (Ultomiris ${ }^{\mathrm{TM}}$; Alexion Pharmaceuticals, Inc., Boston, MA, USA), also administered via intravenous infusion, is the only other approved treatment for PNH [10]; it was designed to cause longer-acting C5 complement inhibition than that of eculizumab (up to 8 weeks vs 2 weeks) [11]. In two phase Ib/II studies, ravulizumab led to reduced complement-mediated hemolysis and improved health-related quality of life (HRQoL) [12]. In two phase III studies, the '301 study' [13] and the '302 study' [14], ravulizumab demonstrated non-inferiority to eculizumab across multiple endpoints, including rate of $\mathrm{BTH}$, in both complement inhibitor-naïve and experienced patients with PNH $[13,14]$. A recent article published by Brodsky et al. in Haematologica [15] investigated data from patients who experienced BTH in the 26-week period of study 301 and 302. Brodsky et al. showed that none of the BTH events experienced by patients treated with ravulizumab in both studies were related to suboptimal free C5 inhibition, while BTH events in patients treated with eculizumab were related to suboptimal free C5 inhibition (7 out of 15 in study 301; four out of seven in study 302). Immediate, complete, and sustained C5 inhibition achieved with ravulizumab reduces the risk of BTH by eliminating BTH associated with suboptimal C5 inhibition in patients with PNH [15]. Fewer
BTH events due to suboptimal free C5 inhibition may lead to improved health outcomes and associated reductions in medical management and administration costs for ravulizumab compared with eculizumab [16].

In addition, ravulizumab is likely to lead to reduced treatment burden compared with eculizumab, owing to its lower infusion frequency. Findings from a discrete choice experiment have demonstrated that given the choice between a series of hypothetical treatments with varying characteristics, patients most prefer treatments with a lower frequency of administration [17].

Further, in a sub-study of one of the phase III studies described above, patient preference data demonstrated that a significantly greater proportion of patients preferred ravulizumab over eculizumab or no preference (93\% vs 7\%, $p<0.001$ ), which was largely owing to its convenient treatment administration and its positive effect on HRQoL (e.g., improvement in ability to plan activities) [18]. To inform a value assessment of ravulizumab vs eculizumab by payers, the objective of this study was to conduct a cost-utility analysis (CUA) of the two treatments in adult patients with $\mathrm{PNH}$, from a US payer perspective.

\section{Methods}

\subsection{Review of Clinical Evidence}

To ascertain the relevant outcomes to include in the CUA of ravulizumab vs eculizumab for the treatment of $\mathrm{PNH}$, discussions were held with clinical experts, and targeted searches of MEDLINE were conducted. This resulted in a list of outcomes relevant to the natural history of PNH [Electronic Supplementary Material (ESM)]. From this list, only outcomes that may differ based on complement inhibitor treatment (i.e., ravulizumab or eculizumab) were considered relevant for potential inclusion in the CUA model. These did not include, for example, thrombosis, renal impairment, and pulmonary hypertension; although PNH treated with supportive care is characterized by these major clinical outcomes, they are effectively managed with eculizumab treatment $[5,8,19-22]$, and their incidence is not expected to differ when treated with ravulizumab vs with eculizumab, based on non-inferiority demonstrated in two clinical studies of ravulizumab [13,14] (further details of these studies are provided in Sect. 2.3.1). Outcomes that remained of relevance to patients treated with eculizumab included BTH, Neisseria meningitidis infection, extravascular hemolysis, underlying bone marrow disorders, and possible effects of eculizumab during pregnancy [23]. Upon further review of clinical evidence, the risks of both $N$. meningitidis infection [24] and complications during pregnancy $[25,26]$ were considered low enough to be excluded. 
Finalization of outcomes and disease pathways to be included in the CUA model was based on feedback from clinical experts in PNH and health economics experts. The following sections describe the methodology and findings of the chosen model.

\subsection{Cost-Utility Analysis Model}

\subsubsection{Overview of Model}

A CUA from a US payer perspective was conducted using a Markov (state-transition) model. The health states of the model were selected based on the evidence review previously described and on feedback from PNH clinical experts. This resulted in a model with 11 health states: eight related to BTH events (with distinction between BTH events related to suboptimal free $\mathrm{C} 5$ inhibition vs related to CAC), two related to mortality (natural/background and PNH-related), and one related to spontaneous remission (Fig. 1).

A BTH event due to suboptimal free C5 inhibition was defined as individual free C5 greater than or equal to $0.5 \mu \mathrm{g} /$ $\mathrm{mL}$, aligning with the definition used in clinical trial data $[13,14]$. A CAC BTH event was defined as any condition (e.g., infection) known to increase complement activity and result in a transient increase in hemolysis [2].

A lifetime horizon was used because PNH is a chronic disease that affects patients with a median age at diagnosis of 35-40 years [27]. A model cycle length of 2 weeks was used to align with visit frequency observed in clinical trial data $[13,14]$ and with the eculizumab administration interval of 2 weeks.

\subsubsection{Comparator}

The comparator in the analysis is eculizumab, the dosing of which differs across the patient cohorts, as described further in Sect. 2.2.3.

\subsubsection{Patient Populations}

Three types of adult PNH patient populations (cohorts) were considered for both the base-case analyses and further scenario analyses, according to patient history of eculizumab dosing prior to treatment with ravulizumab: Cohort 1 , patients naïve to eculizumab treatment (initiating labeled dosing at the start of the model); Cohort 2, patients who

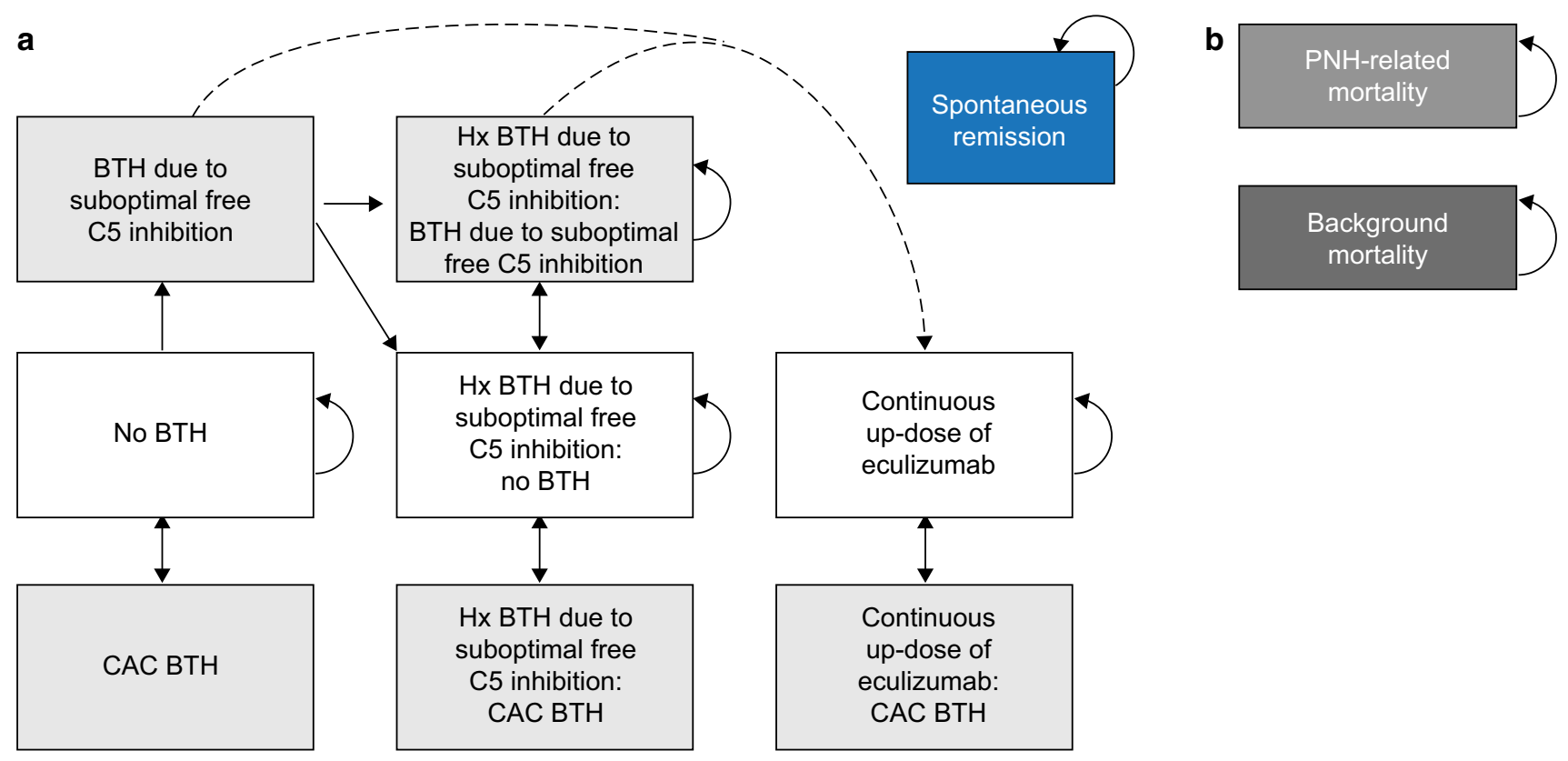

Fig. 1 Illustration of health states used in the cost-utility analysis model. a Living health states. Light grey health states (five boxes shown) indicate that a patient is having a breakthrough hemolysis (BTH) event; white health states indicate that a patient is not having a BTH event. For all BTH states, it is possible to specify that a patient would receive a 'single' up-dose, corresponding to the period of the BTH event. Single up-doses do not affect a patient's clinical progression but incur costs and may be (if specified) associated with a health-utility improvement in the period. The blue health state indi- cates spontaneous remission; patients may transition to this state at any time from living health states. Spontaneous remission occurs based on patient-level data reported in Hillmen et al. [1] b Mortality health states. Patients may transition to background mortality at any time from any living health state. Transitions to paroxysmal nocturnal hemoglobinuria (PNH)-related mortality are only possible from BTH states if an excess mortality risk of BTH is specified. CAC complement-amplifying condition, $H x$ history of 
are clinically stable on the approved maintenance dose of eculizumab (900 mg every 2 weeks); and Cohort 3, patients who are clinically stable on off-label use of a higher maintenance dose of eculizumab (92.5\% on $1200 \mathrm{mg}$ and $7.5 \%$ on $1500 \mathrm{mg}$, every 2 weeks, based on data on file). The overall patient population was allocated to Cohort 1 , Cohort 2, and Cohort 3, according to proportions of $8.2 \%, 91.8 \%$, and $0 \%$, respectively. This was based on the prevalence of $\mathrm{PNH}$ and the incidence of new cases of PNH reported by Hill et al. [28] (see the ESM for more details). Although 0\% of patients were allocated to Cohort 3 , results for this scenario are illustrated to reflect the costs and benefits for patients already requiring a higher dose of eculizumab at baseline.

\subsection{Trial Data and Model Inputs}

\subsubsection{1 and 302 Studies}

The CUA model was based on data from two phase III randomized studies: the 301 study [13] and the 302 study [14]. The 301 study (NCT02946463) is a phase III, openlabel, multicenter study that assessed the non-inferiority of ravulizumab compared with eculizumab in adults with PNH who are complement inhibitor-naïve ( $n=246 ; 125$ on ravulizumab, 121 on eculizumab). It began with a 26 -week randomized treatment period and has a follow-up extension of up to 5 years. At baseline, the study sample had a mean (standard deviation) age of 45.5 (15.7) years and 45.5\% were female. The 302 study (NCT03056040) used the same study design to assess the non-inferiority of ravulizumab compared with eculizumab in patients with $\mathrm{PNH}$ who are clinically stable at the labeled dose of eculizumab $(n=195$; 97 on ravulizumab, 98 on eculizumab). The study sample had a mean (standard deviation) age of 47.7 (14.2) years and $49.7 \%$ were female. In both studies, non-inferiority of ravulizumab vs eculizumab was demonstrated for all endpoints assessed. Although there were no statistically significant differences in the rate of BTH due to suboptimal C5 inhibition, there were fewer events in the ravulizumab group than in the eculizumab group in both the 301 study (zero vs seven events) [13] and the 302 study (zero vs four events) [14, 15].

\subsubsection{Model Inputs}

Model inputs were estimated based on patient visit-level data from the 26-week randomized treatment periods of the two phase III randomized studies described earlier, and supplemented with available evidence from the published literature $[11,12]$. In alignment with definitions of the model cohorts, Cohort 1 outcomes were modeled based on data from the 301 study, Cohort 2 outcomes were modeled based on data from the 302 study, and Cohort 3 outcomes were modeled based on a combination of the evidence from the 301 and
302 studies and assumptions where necessary (as described below). Model inputs were as follows.

- Baseline characteristics of the patient population, including mean age, distribution across modeled cohorts, and information on prior use of eculizumab therapy (for study 302 participants).

- Transition probabilities, to reflect the probability of moving from one health state to another in each cycle of the model. Probabilities were based on patient visit-level data from clinical studies 301 and 302 [13, 14], as well as data from Hillmen et al. [1] for spontaneous remission.

- Eculizumab management practices in response to BTH events, based on current clinical practices in the USA [2, 29].

- Mean blood transfusion requirements, by treatment (ravulizumab vs eculizumab), estimated based on patient visit-level data from clinical studies 301 and 302 [13, 14].

- Health utility, estimated by mapping the HRQoL measure collected in the 301 and 302 studies [European Organization for the Research and Treatment of Cancer Quality of Life Questionnaire (QLQ-C30)] to EuroQol Group's three-level version of EQ-5D (the EQ-5D-3L), a measure commonly used for valuing HRQoL [30]. Mapping was performed using the methodology reported in McKenzie and van der Pol [31].

- Health-utility benefit of reduced healthcare provider visit frequency, estimated using a discrete choice experiment in the UK [17], which isolated the HRQoL impact of visit frequency from other aspects of treatment (ESM).

- Drug costs, based on 2018 wholesale acquisition cost prices per 300-mg vial from the IBM Micromedex RED BOOK database [32].

- Infusion administration costs, based on final 2018 Medicare reimbursement rates for Current Procedural Terminology codes 96413 and 96415 ('Chemotherapy administration, intravenous infusion', first and subsequent hours) [33], applied to estimated administration times stated in US prescribing information for eculizumab and ravulizumab $[3,10]$.

- Costs of other administration-day procedures and meningococcal vaccines, based on an analysis of eculizumab utilization using electronic health records and claims data from 2018 [34].

- Blood transfusion costs, based on payment amounts from Medicare's hospital outpatient payment policies for 2018 (for Healthcare Common Procedure Coding System codes P9038, 86900, 86901, 86902, 86904, 86905, and 36430) [35].

Clinical outcomes beyond the 26-week randomized treatment periods of the clinical studies were extrapolated. In 
particular, it was assumed that transitions to BTH states and spontaneous remission continued to occur, based on the transition probabilities modeled in the first 26 weeks. An exception to this was the transition to a first BTH event due to suboptimal free C5 inhibition for Cohort 1 patients, which was assumed to become equal to the corresponding probability for Cohort 2 patients after 26 weeks (owing to the fact that Cohort 1 patients who had not experienced a BTH event due to suboptimal free C5 inhibition by 26 weeks after eculizumab initiation at labeled dosing took the profile of a patient at baseline of the 302 study at 26 weeks). Extrapolation of the same transition probability to future model cycles implies that there is a constant hazard of the transition over time (i.e., that time to the event follows an exponential distribution), as is characteristic of Markov models. Values for all model inputs, for base-case and sensitivity analyses, are provided in Table 1 (further details of inputs are also provided in the ESM).

\subsection{Outcome Measures}

Discounted lifetime costs and benefit were calculated for patients when treated with eculizumab and when treated with ravulizumab. Benefit was measured as the number of quality-adjusted life-years (QALYs) accrued following the start of treatment; QALYs were computed by multiplying years of life by health-utility values. The incremental costs and benefit, and incremental cost-effectiveness ratios (ICERs) reflecting cost per QALY gained, were computed for ravulizumab vs eculizumab. Results are presented overall and by patient cohort. A standard US annual discount rate of 3.0\% per annum was applied to costs and QALYs [36].

For each modeled cohort in the base-case analysis, Markov trace plots were created showing the proportion of patients belonging to each health state over the model's lifetime horizon. In addition, line plots were generated comparing the per-patient per-year drug and administration costs for ravulizumab and eculizumab, for patients remaining on treatment; this plot, for the overall population, is presented in Fig. 2.

\subsection{Base-Case Analysis}

\subsubsection{Assumptions Applying to All Patient Types}

The following assumptions concerning health states and treatment were made across all patient cohorts in the base-case analysis. Values of model inputs are provided in Table 1.

- Treatment of BTH events due to suboptimal free C5 inhibition was eculizumab with continuous up-dosing [2, 29].
- Treatment of CAC BTH events depended on what the treatment was at the time of the BTH event.

- Patients on labeled dosing of eculizumab received up-dosing of $300 \mathrm{mg}$ (one vial) of eculizumab for a single cycle ('single up-dosing').

- Patients who were already continuously up-dosed on eculizumab were given no further up-dosing.

- Symptoms of BTH events due to suboptimal free C5 inhibition lasted for 2 days before being effectively managed.

- Persistence of BTH events due to suboptimal free C5 inhibition aligned with data from the 301 and 302 studies $[13,14]$.

- Patients who achieved spontaneous remission stopped PNH-related treatment (including complement inhibitor therapy).

- Patients treated with eculizumab and ravulizumab had the same rates of spontaneous remission.

- BTH events were not associated with an increased mortality risk above the background mortality of the general population. This was a conservative assumption, despite evidence that indicates an excess mortality associated with elevated lactate dehydrogenase (i.e., associated with BTH events, as elevated lactate dehydrogenase was incorporated into the BTH definition used in the 301 and 302 studies) [37]. Evidence from Kelly et al. [8] found that patients whose BTH events are managed with continuous eculizumab up-dosing have comparable survival to that of the general population $[9,38]$. Further, various studies have shown that treatment with eculizumab improves survival and reduces the risk of hemolysis [5, $8,39]$.

\subsubsection{Assumptions Specific to Patient Cohorts}

Patients in Cohort 1 had a time-dependent change in the risk of an initial BTH event due to suboptimal free C5 inhibition, such that the transition probability associated with having this event decreased from the beginning of treatment to 26 weeks later. This reflects the fact that Cohort 1 patients took the profile of a Cohort 2 patient at 26 weeks [14].

Patients in Cohort 3 were assumed not to be at risk of BTH due to suboptimal free C5 inhibition, as they were either up-dosed on eculizumab or were receiving ravulizumab. The lack of risk of BTH events due to suboptimal free C5 inhibition when treated with ravulizumab is based on findings from the 301 study showing that patients who experienced BTH events due to suboptimal free $\mathrm{C} 5$ inhibition while receiving eculizumab in the first 26 weeks did not experience any BTH events due to suboptimal free C5 inhibition when they switched to ravulizumab between weeks 26 and 52 [13]. Otherwise, these patients were assumed to 


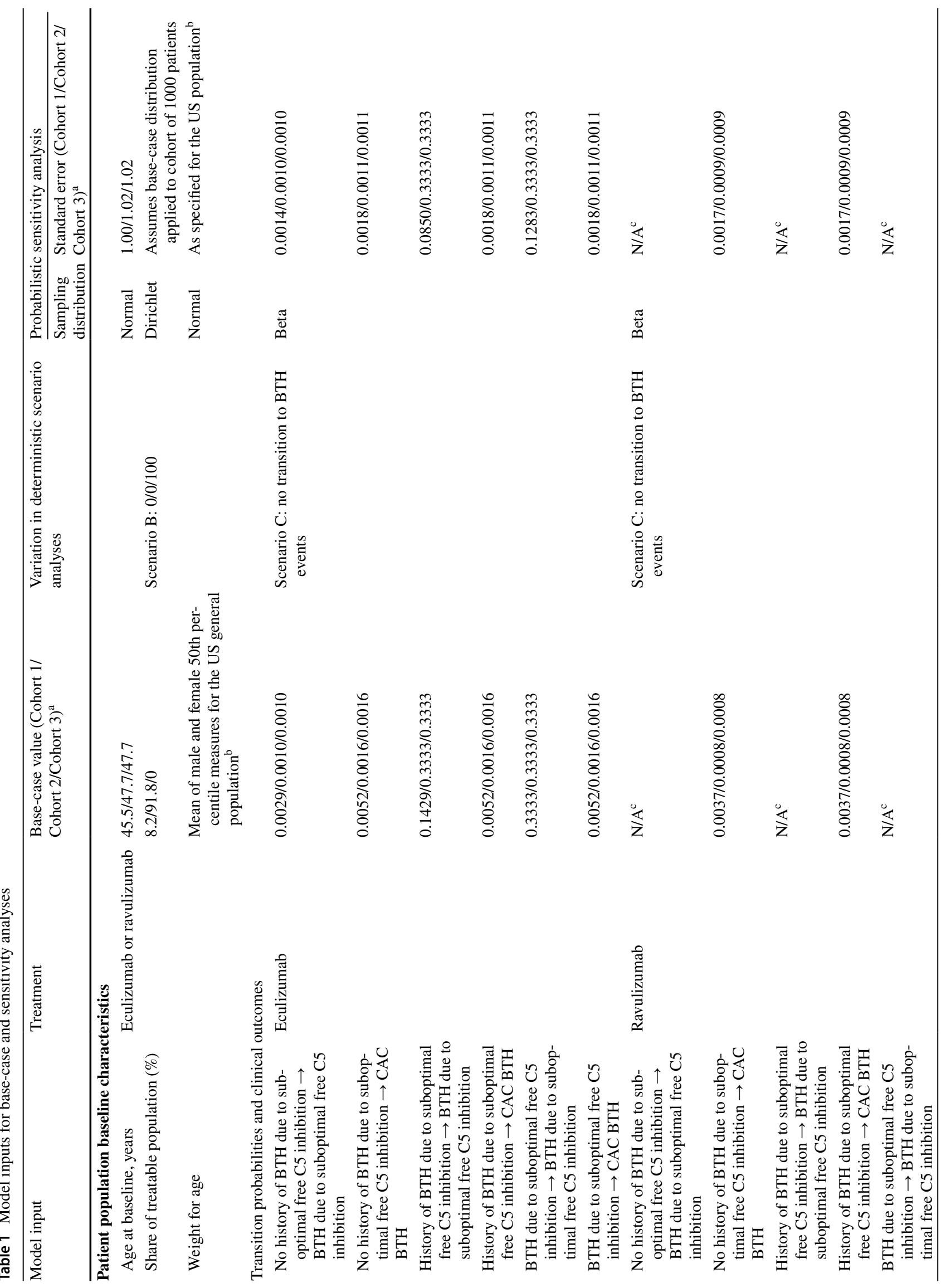




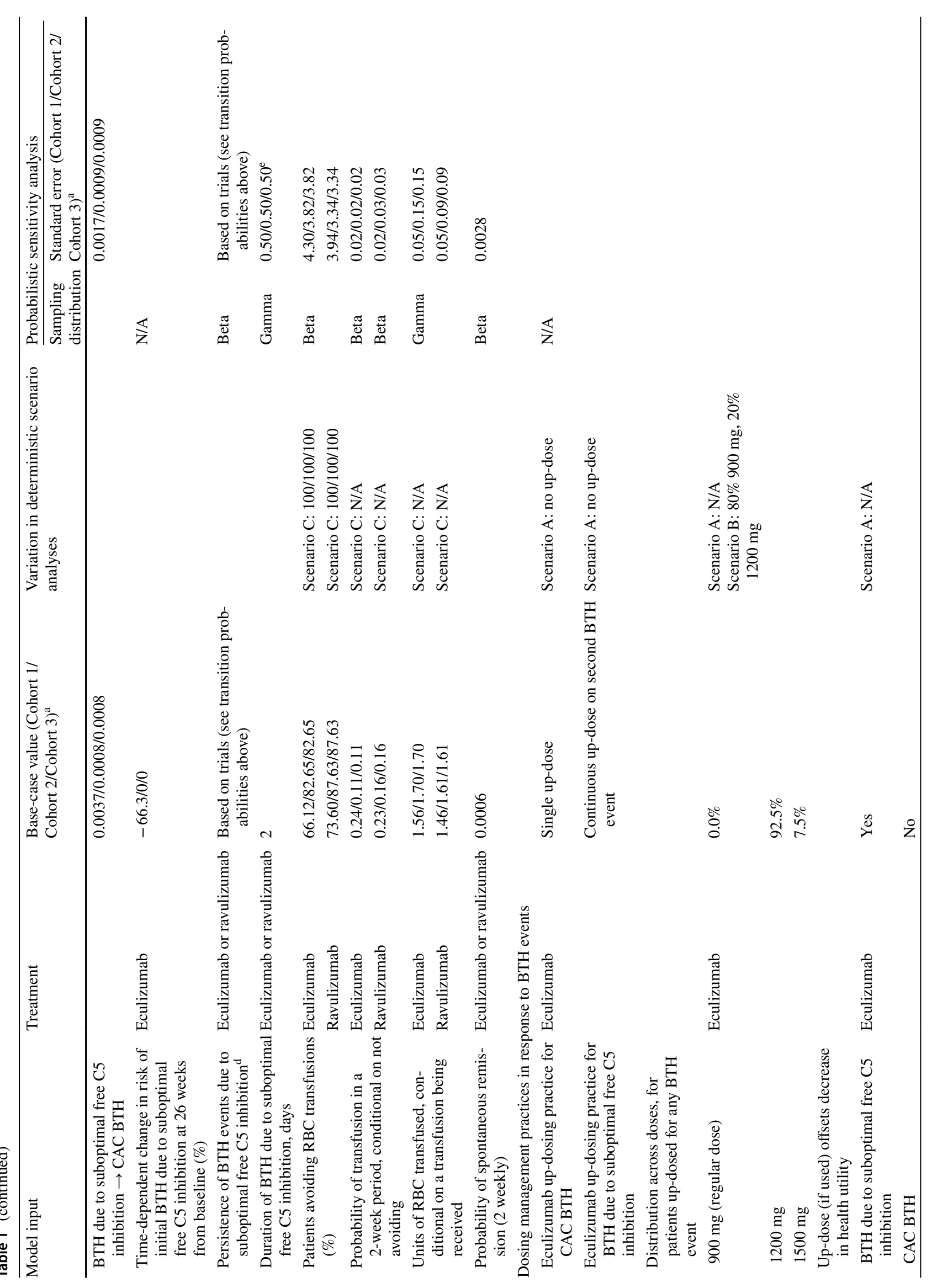




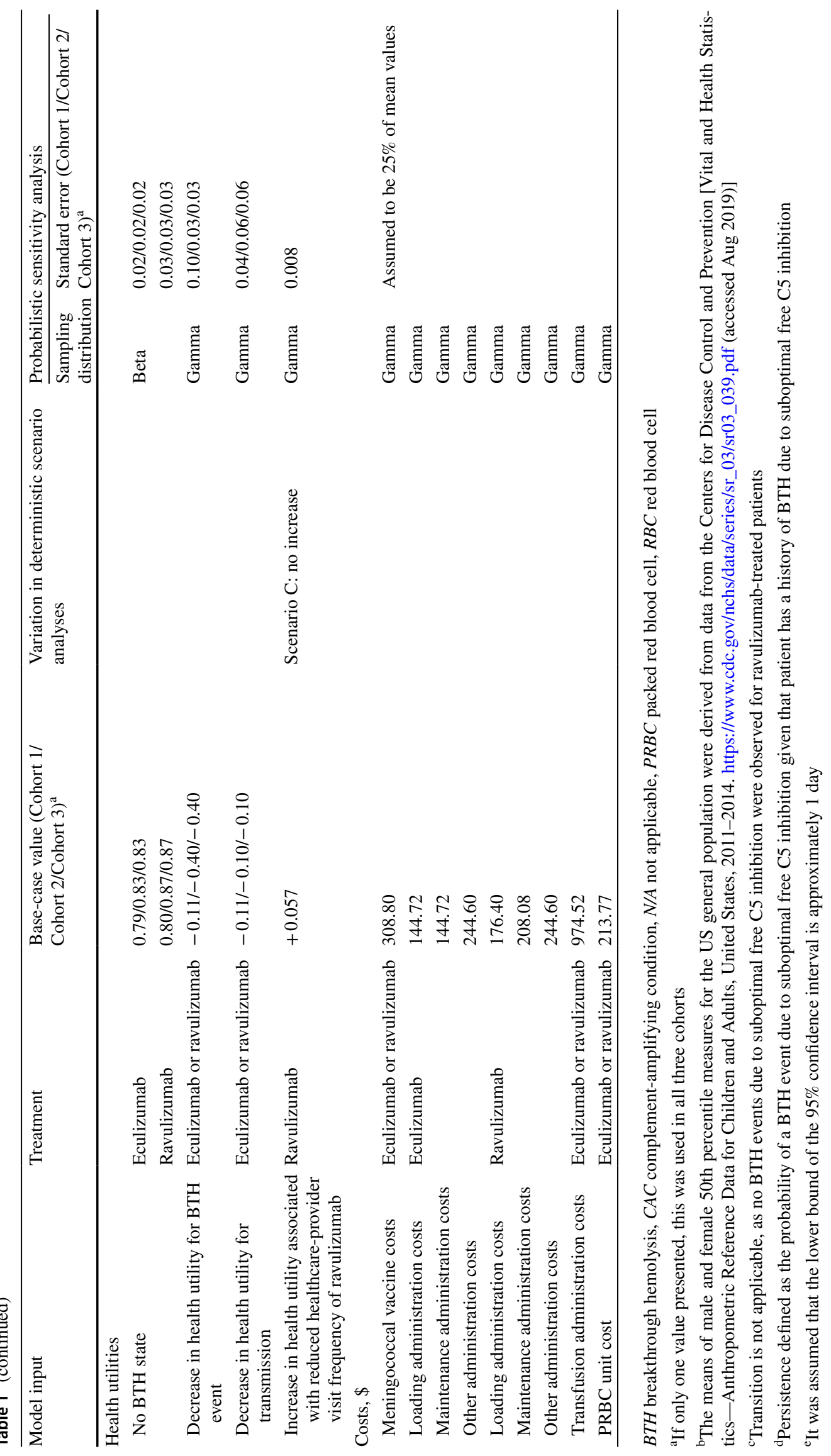


experience the same outcomes as patients in Cohort 2, given their shared history of eculizumab therapy.

\subsection{Sensitivity Analyses}

\subsubsection{Deterministic Scenario Analyses}

In addition to the base-case analysis of the CUA, three alternative scenarios were tested to assess the impact of plausible variation of key model inputs and assumptions.

- Scenario A, no eculizumab dosing adjustment. This scenario assumed that clinical management practices did not adjust eculizumab dosing or administration frequency in response to BTH events.

- Scenario B, real-world dosing. In this scenario, an increase in dose of eculizumab was not triggered by BTH incidence; instead, dosing of eculizumab reflected real-world evidence from the UK that $20 \%$ of patients require a higher maintenance dose than the labeled dose [9] (conservatively assumed to be the lowest dose higher than the labeled 900-mg dose, i.e., $1200 \mathrm{mg}$ ).

- Scenario C, non-inferiority. This scenario assumed no difference in the risk of BTH events and transfusions between eculizumab and ravulizumab, based on the evidence of non-inferiority between the treatments from the 301 and 302 studies [13, 14]. This scenario also assumed that there was no visit frequency-related utility benefit (as opposed to the base case) for ravulizumab. Consequently, in this scenario, the benefit of ravulizumab vs eculizumab is limited to the mean difference in mapped utilities between treatment arms in the clinical trials [13, 14], controlling for (i.e., excluding benefit of) BTH, transfusions, and healthcare provider visit frequency.

Model inputs associated with these scenario analyses are provided in Table 1, and further details are provided in the ESM.

\subsubsection{Probabilistic Sensitivity Analysis}

A Monte Carlo simulation of the model was carried out to incorporate parameter uncertainty. This involved running 1000 iterations of the model, within which random values of model parameters were drawn from associated statistical distributions (Table 1). Incremental costs and benefit (ravulizumab minus eculizumab) were computed under each iteration.

\section{Results}

\subsection{Base-Case Analysis}

In the base-case analysis for the overall population (the weighted average of patient cohorts), total costs were lower and health benefit (number of QALYs) was greater for ravulizumab than for eculizumab (Table 2); incremental costs were $-\$ 1,673,465$ and incremental QALYs were 1.67 for ravulizumab vs eculizumab. The resulting negative ICER, $-\$ 1,000,818$, indicates that the gain of one QALY is achieved with associated savings of $\$ 1,000,818$ by treating patients with ravulizumab rather than eculizumab. In the other patient populations, Cohorts 1-3, there were also cost savings, increased benefit, and negative ICERs (Table 2). Markov trace plots are illustrated in Figs. 1-3 of the ESM.

In the examination of maintenance costs over time, mean annual maintenance-year costs of ravulizumab were lower than for eculizumab after treatment initiation. Eculizumab maintenance-year costs continued to increase over time, with the increase in the number of patients continuously up-dosed owing to the incidence of BTH over time, whereas mean annual maintenance-year ravulizumab costs remained stable (Fig. 2). Figure 2 illustrates the bimodal variation in perpatient per-year cost for ravulizumab (given that six or seven administrations may be required in alternating maintenance years), and the growth over time of eculizumab drug costs with an increasing share of patients receiving a higher dose for management of BTH events.

\subsection{Deterministic Scenario Analyses}

In scenario analyses, incremental costs (ravulizumab minus eculizumab) had a range from $-\$ 893,537$ (scenario C) to - \$1,448,943 (scenario B) and incremental benefit had a range from +0.72 QALYs (scenario $\mathrm{C}$ ) to +1.75 QALYs (scenario A). These cost savings and increased benefit resulted in negative and favorable ICERs for ravulizumab vs eculizumab in all cases (Table 3 ).

\subsection{Probabilistic Sensitivity Analyses}

Simulation of the model showed that incremental costs and benefit clustered around negative costs and positive benefit, indicating consistent cost savings and increased benefit of ravulizumab vs eculizumab over repeated iterations (Fig. 3). For the overall population, in $99.9 \%$ of iterations of the probabilistic sensitivity analysis, total costs were lower and health benefit (number of QALYs) was greater for ravulizumab than for eculizumab; therefore, the cost-effectiveness acceptability curve of ravulizumab vs eculizumab would lie at $99.9 \%$ or above for all positive ICER thresholds. 
Fig. 2 Mean annual complement inhibitor drug and administration costs over time: overall study population



\begin{tabular}{|c|c|c|c|c|}
\hline Study population & Cost/benefit measure & Eculizumab & Ravulizumab & $\begin{array}{l}\text { Incremental (ravuli- } \\
\text { zumab minus eculi- } \\
\text { zumab) }\end{array}$ \\
\hline \multirow[t]{5}{*}{ Overall population } & Medical costs, US\$ & 13,512 & 12,771 & -742 \\
\hline & Drug costs, US\$ & $9,350,355$ & $7,677,632$ & $-1,672,724$ \\
\hline & Total costs, US\$ & $9,363,868$ & $7,690,403$ & $-1,673,465$ \\
\hline & Number of QALYs & 17.25 & 18.93 & 1.67 \\
\hline & ICER, US\$ ${ }^{\mathrm{a}}$ & $-1,000,818$ & & \\
\hline \multirow[t]{5}{*}{ Cohort $1(8 \%)^{\mathrm{b}}$} & Medical costs, US\$ & 46,949 & 34,143 & $-12,806$ \\
\hline & Drug costs, US\$ & $9,655,970$ & $7,864,207$ & $-1,791,763$ \\
\hline & Total costs, US\$ & $9,702,919$ & $7,898,350$ & $-1,804,568$ \\
\hline & Number of QALYs & 16.87 & 18.07 & 1.19 \\
\hline & ICER, US\$ $\$^{\mathrm{a}}$ & $-1,512,000$ & & \\
\hline \multirow[t]{5}{*}{ Cohort $2(92 \%)^{b}$} & Medical costs, US\$ & 10,535 & 10,868 & 333 \\
\hline & Drug costs, US\$ & $9,323,143$ & $7,661,019$ & $-1,662,124$ \\
\hline & Total costs, US\$ & $9,333,678$ & $7,671,887$ & $-1,661,792$ \\
\hline & Number of QALYs & 17.29 & 19.00 & 1.71 \\
\hline & ICER, US\$ ${ }^{\mathrm{a}}$ & $-969,137$ & & \\
\hline \multirow[t]{5}{*}{ Cohort $3(0 \%)^{\mathrm{b}}$} & Medical costs, US\$ & 10,535 & 10,868 & 333 \\
\hline & Drug costs, US\$ & $11,555,779$ & $7,661,019$ & $-3,894,760$ \\
\hline & Total costs, US\$ & $11,566,315$ & $7,671,887$ & $-3,894,428$ \\
\hline & Number of QALYs & 17.29 & 19.00 & 1.71 \\
\hline & ICER, US\$ $\$^{\mathrm{a}}$ & $-2,272,060$ & & \\
\hline
\end{tabular}

ICER incremental cost-effectiveness ratio, $Q A L Y$ quality-adjusted life-year

${ }^{a}$ ICERs show cost per QALY gained, ravulizumab vs eculizumab

${ }^{b}$ Percentages indicate the distribution of cohorts comprising the overall study population

\section{Discussion}

In this CUA from a US payer perspective, the costs and benefit of treatment with ravulizumab vs eculizumab in adult patients with PNH were assessed using a Markov model, an approach commonly used for CUA to inform reimbursement policies for new interventions, which distils decision problems into a finite number of conceptual states, facilitating communication and interpretation [40]. Ravulizumab was found to have economic dominance (i.e., cost savings and health benefit) compared with eculizumab in the treatment of adults with PNH. Following the initiation year, treatment with ravulizumab was associated with substantially lower costs than treatment with eculizumab, and savings associated with ravulizumab grew when considering eculizumab dosing responses that may be used to manage $\mathrm{BTH}$ events due to 
Table 3 Deterministic scenario analyses: incremental costs and benefit of ravulizumab vs eculizumab under three scenarios; overall study population

\begin{tabular}{|c|c|c|c|}
\hline \multirow[t]{2}{*}{ Scenario } & \multicolumn{2}{|c|}{$\begin{array}{l}\text { Incremental measures } \\
\text { (ravulizumab minus } \\
\text { eculizumab) }\end{array}$} & \multirow[t]{2}{*}{ ICER, US\$ $\$^{\mathrm{a}}$} \\
\hline & $\begin{array}{l}\text { Num- } \\
\text { ber of } \\
\text { QALYs }\end{array}$ & Costs, US\$ & \\
\hline Base case & 1.67 & $-1,673,465$ & $-1,000,818$ \\
\hline $\begin{array}{l}\text { Scenario A: no eculizumab } \\
\text { dosing adjustment }\end{array}$ & 1.75 & $-894,279$ & $-512,128$ \\
\hline $\begin{array}{l}\text { Scenario B: real-world dos- } \\
\text { ing }\end{array}$ & 1.71 & $-1,448,943$ & $-845,332$ \\
\hline Scenario C: non-inferiority & 0.72 & $-893,537$ & $-1,240,186$ \\
\hline
\end{tabular}

ICER incremental cost-effectiveness ratio, $Q A L Y$ quality-adjusted life-year

${ }^{\mathrm{a}}$ ICERs show cost per QALY gained, ravulizumab vs eculizumab

suboptimal free C5 inhibition. In addition, ravulizumab was found to provide an additional moderate health benefit to the patient compared with eculizumab.

The main driver of cost savings was the reduction in maintenance-year complement inhibitor drug costs. After treatment initiation, mean annual maintenance-year costs of ravulizumab were at least $10 \%$ lower than those of eculizumab. Eculizumab maintenance-year costs grew over the model's time horizon, owing to a cumulative incidence of BTH events and resulting up-dosing and associated higher drug costs. The improved benefit was due to a reduction in treatment burden, as well as improved outcomes.

The strengths of the study include the process employed to define the conceptual structure of the CUA model (aligning with good practices for modeling [41]), as well as the estimation of its inputs and parameters. The model structure reflects feedback from PNH clinical experts on the major differentiators of costs and HRQoL between ravulizumab and eculizumab. Input parameters for the model were estimated from patient visit-level data from phase III clinical studies $[13,14]$. These included rates and persistence of BTH events due to suboptimal free C5 inhibition, blood transfusion requirements, and utility values (from HRQoL outcomes). Sensitivity analysis involving repeated simulation of the model incorporated uncertainty in the model's parameters, and showed that there were substantial cost savings overall; findings from deterministic scenario analyses were consistent with the base-case model. In reporting the results of this CUA, we adhered to the CHEERS checklist, which outlines ISPOR's good reporting practice guidelines for health economic evaluations [42].

A primary limitation of the study is that extrapolation of clinical outcomes was required beyond the data from the 26-week randomized treatment periods of the clinical studies $[13,14]$. Furthermore, owing to the fact that eculizumab has only been approved for PNH since 2007, evidence against which to validate over a lifetime model horizon is not available. Nevertheless, the CUA's predictions of cumulative incidence of BTH due to suboptimal free C5 inhibition over time broadly align with available evidence from patients treated with eculizumab. For example, in a study by Hillmen et al. [5] in 140 patients whose plasma concentrations of eculizumab and hemolytic activity were reviewed, $10 \%$ were observed to have experienced multiple occurrences of trough eculizumab concentrations less than $35 \mu \mathrm{g} / \mathrm{mL}$ (indicating a BTH event due to suboptimal free $\mathrm{C} 5$ inhibition) at a median follow-up of approximately 2.5 years. Our model outcomes align with Hillmen et al.'s findings, considering that the Markov traces for Cohort 1 indicated that, at 2.5 years from baseline, $5-10 \%$ of patients had experienced BTH due to suboptimal free C5 inhibition. Furthermore, evidence from patients treated in Leeds, UK shows that the
Fig. 3 Distribution of costs and quality-adjusted life-years (QALYs) accounting for the combined impact of parameter uncertainty in repeated simulations: overall study population. Each point indicates an iteration of the model simulation (1000 iterations)

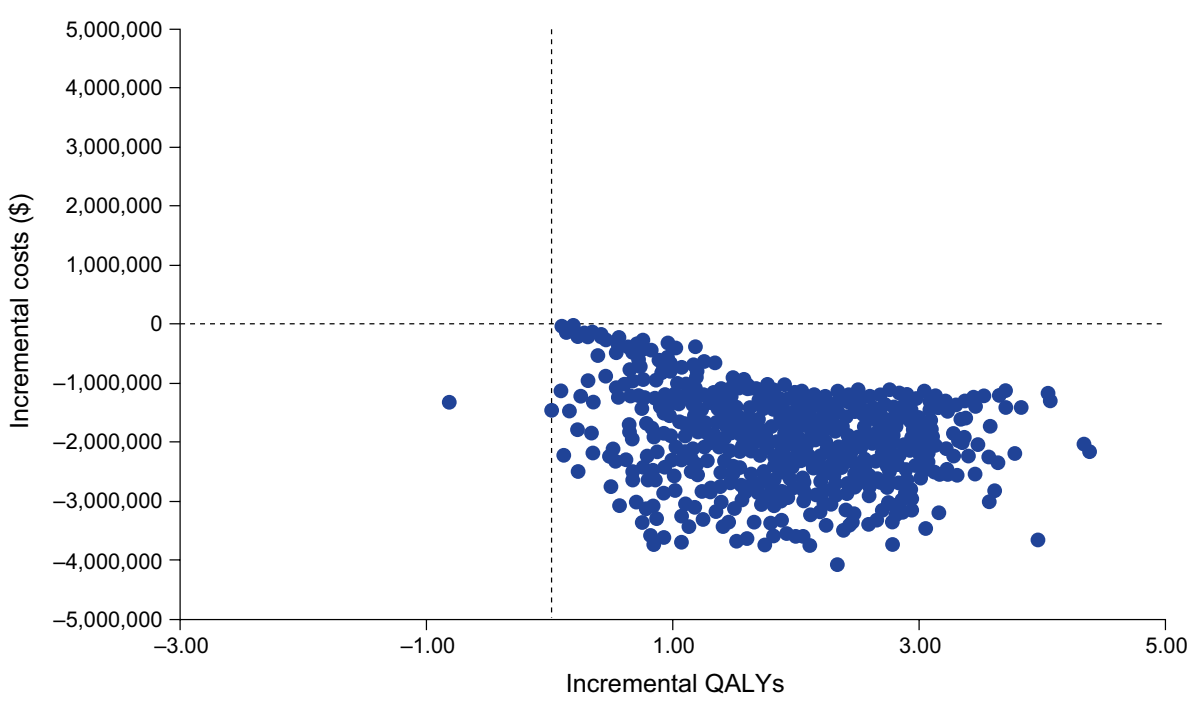


share of patients receiving an increase in their dose of eculizumab (a higher dose being a proxy for those with a history of BTH events, given treatment practices in the UK) has grown over time, similar to modeled outcomes in the CUA [8]. In a 2011 publication on the Leeds patient population, 5 of 74 patients $(7 \%)$ were recorded as receiving a higher dose of eculizumab, whereas in 2017 findings of this study sample, more than $20 \%$ of patients required a higher dose of eculizumab [9]. This result suggests that, at a maximum possible follow-up of approximately 12 years, $20 \%$ of patients may experience suboptimal free C5 inhibition. Again, the results of our CUA align with these data, in that the Markov traces for Cohort 1 indicated that, at 12 years from baseline, approximately $20 \%$ of patients have experienced a BTH event due to suboptimal free $\mathrm{C} 5$ inhibition.

The difference between rates of BTH due to suboptimal C5 inhibition was a key difference in health outcomes between treatments in our model. This difference relied on data from the 301 study [13] and the 302 study [14, 15] which showed a numerically lower rate of BTH due to suboptimal C5 inhibition in the ravulizumab group than in the eculizumab group, but not a statistically significant lower rate. Statistical significance can be difficult to demonstrate in studies of rare diseases, owing to the low incidence of clinical events, as was the case for BTH in both studies. However, following discussion on this topic at a $2018 \mathrm{PNH}$ advisory board meeting [38], experts from the National Institute of Health and Care Excellence and the Scottish Medicines Consortium advised that modeling of numerical differences in the base case is an acceptable approach, provided a probabilistic sensitivity analysis is conducted. In the current paper, we have included a probabilistic sensitivity analysis, and we have also investigated the scenario in which there is no difference in rates of BTH due to suboptimal C5 inhibition between the treatments.

Despite aligning with good practices for decision-analytic modeling, the CUA also has certain other limitations. Health-state utility values were determined based on mapping the European Organization for the Research and Treatment of Cancer Quality of Life Questionnaire QLQ-C30 to the EQ-5D-based utilities, owing to lack of availability of EQ-5D data. The appropriateness of a mapping algorithm may vary depending on differences between the population it was derived from and the population to which it is applied. However, the mapping algorithm used (McKenzie and van der Pol [31]) was recommended in a review of such mappings [43]. Additionally, certain outcomes of relevance to patients with PNH receiving eculizumab, including extravascular hemolysis, iron overload, and underlying bone marrow disorders, were excluded from the CUA. However, their exclusion is not expected to alter incremental results significantly because there is no evidence to suggest a difference in these outcomes between ravulizumab and eculizumab; consequently, HRQoL implications of the outcomes would be the same across treatments, and survival impacts (primarily relating to bone marrow disorders) are rare [44].

\section{Conclusions}

Findings from this CUA, which was informed by clinical experts and evidence from randomized studies, demonstrate that, compared with eculizumab, ravulizumab provides a large cost saving from a US payer perspective, as well as an increased benefit to adult patients with PNH.

Acknowledgments The authors thank Dr. Anita Hill, Consultant Haematologist for Leeds Teaching Hospitals NHS Trust, Leeds, UK, and Lead Consultant for the National PNH Service, Leeds, UK, at the time of the study (current affiliation: employee of Alexion Pharmaceuticals, Inc.) and Dr. Austin Kulasekararaj, Consultant Haematologist, King's College Hospital, London, UK, for providing clinical expertise to develop the model in this study. Editorial review was provided by Shweta Rane, PhD, CMPP of Alexion Pharmaceuticals, Inc. Lisa Law (https://orcid.org/0000-0002-9837-6609) of Oxford PharmaGenesis, Oxford, UK, provided medical writing support, which has been funded by Alexion Pharmaceuticals, Inc. in accordance with Good Publication Practice 3.

Author contributions All authors contributed to the conception, design, and interpretation of the cost-utility analysis. TO, MB, SJ, and LT carried out the analysis. All authors contributed to the drafting of the paper or to revising it critically for intellectual content. All authors gave final approval of the version to be published and agree to be accountable for all aspects of the work.

\section{Compliance with Ethical Standards}

Funding This study was funded by Alexion Pharmaceuticals Inc. Alexion Pharmaceuticals Inc. were involved in the conception, design, and interpretation of the findings. They were not involved in conducting the analysis.

Conflict of interest Ioannis Tomazos and Simu K. Thomas are employees of Alexion Pharmaceuticals Inc. Thomas O'Connell, Marric Buessing, Scott Johnson, and Lufei Tu are employees of Medicus Economics, which received funding from Alexion Pharmaceuticals Inc. to participate in this research.

Ethics approval This article does not contain any studies with human participants or animals performed by any of the authors.

Data availability Some of the data generated during and/or analyzed during the current study are not publicly available but are available from the corresponding author on request.

Open Access This article is licensed under a Creative Commons Attribution-NonCommercial 4.0 International License, which permits any non-commercial use, sharing, adaptation, distribution and reproduction in any medium or format, as long as you give appropriate credit to the original author(s) and the source, provide a link to the Creative Commons licence, and indicate if changes were made. The images or other third party material in this article are included in the article's Creative 
Commons licence, unless indicated otherwise in a credit line to the material. If material is not included in the article's Creative Commons licence and your intended use is not permitted by statutory regulation or exceeds the permitted use, you will need to obtain permission directly from the copyright holder. To view a copy of this licence, visit http://creativecommons.org/licenses/by-nc/4.0/.

\section{References}

1. Hillmen P, Lewis SM, Bessler M, Luzzatto L, Dacie JV. Natural history of paroxysmal nocturnal hemoglobinuria. N Engl J Med. 1995;333(19):1253-8. https://doi.org/10.1056/NEJM199511 093331904.

2. Brodsky RA. Paroxysmal nocturnal hemoglobinuria. Blood. 2014;124(18):2804-11. https://doi.org/10.1182/blood-2014-02522128.

3. US Food and Drug Administration. Soliris: full prescribing information; 2007. https://www.accessdata.fda.gov/drugsatfda_docs/ label/2017/125166s422lbl.pdf. Accessed Jun 2019.

4. Höchsmann B, Lee JW, Kulagin A, Hillmen P, Wilson A, et al. Effect of eculizumab in paroxysmal nocturnal hemoglobinuria (PNH) patients with or without high disease activity: results from the International PNH Registry. EHA Learning Center. 207; 181785 .

5. Hillmen P, Muus P, Roth A, Elebute MO, Risitano AM, Schrezenmeier $\mathrm{H}$, et al. Long-term safety and efficacy of sustained eculizumab treatment in patients with paroxysmal nocturnal haemoglobinuria. Br J Haematol. 2013;162(1):62-73. https://doi. org/10.1111/bjh.12347.

6. Nakayama H, Usuki K, Echizen H, Ogawa R, Orii T. Eculizumab dosing intervals longer than 17 days may be associated with greater risk of breakthrough hemolysis in patients with paroxysmal nocturnal hemoglobinuria. Biol Pharm Bull. 2016;39(2):2858. https://doi.org/10.1248/bpb.b15-00703.

7. Peffault de Latour R, Fremeaux-Bacchi V, Porcher R, Xhaard A, Rosain J, Castaneda DC, et al. Assessing complement blockade in patients with paroxysmal nocturnal hemoglobinuria receiving eculizumab. Blood. 2015;125(5):775-83. https://doi.org/10.1182/ blood-2014-03-560540.

8. Kelly RJ, Hill A, Arnold LM, Brooksbank GL, Richards SJ, Cullen M, et al. Long-term treatment with eculizumab in paroxysmal nocturnal hemoglobinuria: sustained efficacy and improved survival. Blood. 2011;117(25):6786-92. https://doi.org/10.1182/ blood-2011-02-333997.

9. Newton DJ, McKinley CE, Ricardo A, Hoarty MD, Arnold L, Riley K, et al. Assessment of eculizumab and C5 levels in PNH treatment: molar ratio of eculizumab to $\mathrm{C} 5$ level effectively predicts ongoing dosage changes in a cohort of 50 patients treated with eculizumab. Blood. 2017;130(Suppl. 1):3484.

10. US Food and Drug Administration. Ultomiris: full prescribing information; 2018. https://www.accessdata.fda.gov/drugsatfda _docs/label/2018/761108s000lbl.pdf. Accessed June 2019.

11. Sheridan D, Yu ZX, Zhang Y, Patel R, Sun F, Lasaro MA, et al. Design and preclinical characterization of ALXN1210: a novel anti-C5 antibody with extended duration of action. PLoS ONE. 2018;13(4):e0195909. https://doi.org/10.1371/journal.pone.01959 09.

12. Roth A, Rottinghaus ST, Hill A, Bachman ES, Kim JS, Schrezenmeier H, et al. Ravulizumab (ALXN1210) in patients with paroxysmal nocturnal hemoglobinuria: results of 2 phase $1 \mathrm{~b} / 2$ studies. Blood Adv. 2018;2(17):2176-85. https://doi.org/10.1182/blood advances.2018020644.

13. Lee JW, Sicre de Fontbrune F, Wong Lee Lee L, Pessoa V, Gualandro S, Fureder W, et al. Ravulizumab (ALXN1210) vs eculizumab in adult patients with PNH naive to complement inhibitors: the 301 study. Blood. 2019;133(6):530-9. https://doi. org/10.1182/blood-2018-09-876136.

14. Kulasekararaj AG, Hill A, Rottinghaus ST, Langemeijer S, Wells R, Gonzalez-Fernandez FA, et al. Ravulizumab (ALXN1210) vs eculizumab in C5-inhibitor-experienced adult patients with $\mathrm{PNH}$ : the 302 study. Blood. 2019;133(6):540-9. https://doi.org/10.1182/ blood-2018-09-876805.

15. Brodsky RA, Peffault de Latour R, Rottinghaus ST, Roth A, Risitano AM, Weitz IC, et al. Characterization of breakthrough hemolysis events observed in the phase 3 randomized studies of ravulizumab versus eculizumab in adults with paroxysmal nocturnal hemoglobinuria. Haematologica. 2020. https://doi.org/10.3324/ haematol.2019.236877.

16. Tomazos I, Sierra R, Cheung A, Brodsky R, Weitz IC, Johnston $\mathrm{K}$. Cost burden of breakthrough hemolysis in patients with paroxysmal nocturnal hemoglobinuria on eculizumab treatment. Value Health. 2019;22(S2):PSY12.

17. Lloyd AJ, Gallop K, Ali S, Myren KJ, Sierra JR, Anokhina K, et al. Preference weights for quality-adjusted life-years estimation for treatments of paroxysmal nocturnal hemoglobinuria in the United Kingdom. Value Health. 2019;22(Suppl. 3):S902. https:// doi.org/10.1016/j.jval.2019.09.2637.

18. Peipert JD, Kulasekararaj A, Gaya A, Langemeijer SMC, Yount S, Ataulfo Gonzalez Fernandez F, et al. Patient preferences for the treatment of paroxysmal nocturnal hemoglobinuria: results of a patient survey of ravulizumab (ALXN1210) and eculizumab [abstract no. PF734]. Poster presented at the 24th EHA Congress; June 13-16, 2019, Amsterdam.

19. Brodsky RA, Young NS, Antonioli E, Risitano AM, Schrezenmeier $\mathrm{H}$, Schubert J, et al. Multicenter phase 3 study of the complement inhibitor eculizumab for the treatment of patients with paroxysmal nocturnal hemoglobinuria. Blood. 2008;111(4):1840 7. https://doi.org/10.1182/blood-2007-06-094136.

20. Hillmen P, Elebute M, Kelly R, Urbano-Ispizua A, Hill A, Rother $\mathrm{RP}$, et al. Long-term effect of the complement inhibitor eculizumab on kidney function in patients with paroxysmal nocturnal hemoglobinuria. Am J Hematol. 2010;85(8):553-9. https://doi. org/10.1002/ajh.21757.

21. Hillmen P, Muus P, Duhrsen U, Risitano AM, Schubert J, Luzzatto L, et al. Effect of the complement inhibitor eculizumab on thromboembolism in patients with paroxysmal nocturnal hemoglobinuria. Blood. 2007;110(12):4123-8. https://doi.org/10.1182/ blood-2007-06-095646.

22. Hillmen P, Young NS, Schubert J, Brodsky RA, Socie G, Muus P, et al. The complement inhibitor eculizumab in paroxysmal nocturnal hemoglobinuria. N Engl J Med. 2006;355(12):1233-43. https ://doi.org/10.1056/NEJMoa061648.

23. Kelly R, Richards S, Hillmen P, Hill A. The pathophysiology of paroxysmal nocturnal hemoglobinuria and treatment with eculizumab. Ther Clin Risk Manag. 2009;5:911-21. https://doi. org/10.2147/tcrm.s3334.

24. Rappuoli R, Pizza M, Masignani V, Vadivelu K. Meningococcal $B$ vaccine $(4 \mathrm{CMenB})$ : the journey from research to real world experience. Expert Rev Vaccines. 2018;17(12):1111-21. https:// doi.org/10.1080/14760584.2018.1547637.

25. Kelly RJ, Hochsmann B, Szer J, Kulasekararaj A, de Guibert S, Roth A, et al. Eculizumab in pregnant patients with paroxysmal nocturnal hemoglobinuria. N Engl J Med. 2015;373(11):1032-9. https://doi.org/10.1056/NEJMoa1502950.

26. Sarno L, Tufano A, Maruotti GM, Martinelli P, Balletta MM, Russo D. Eculizumab in pregnancy: a narrative overview. J Nephrol. 2019;32(1):17-25. https://doi.org/10.1007/s4062 0-018-0517-z.

27. John Hopkins Medicine. Paroxysmal nocturnal hemoglobinuria (PNH). https://www.hopkinsmedicine.org/kimmel_cancer_center/ 
types_cancer/paroxysmal_nocturnal_hemoglobinuria_PNH.html. Accessed June 2019.

28. Hill A, Platts PJ, Smith A, Richards SJ, Cullen MJ, Hill QA, et al. The incidence and prevalence of paroxysmal nocturnal hemoglobinuria $(\mathrm{PNH})$ and survival of patients in Yorkshire. Blood. 2006;108(11).

29. Brodsky RA. Treatment and prognosis of paroxysmal nocturnal hemoglobinuria; 2018. https://www.uptodate.com/contents/treat ment-and-prognosis-of-paroxysmal-nocturnal-hemoglobinuria. Accessed June 2019.

30. EuroQol. EQ-5D-3L. https://euroqol.org/eq-5d-instruments/ eq-5d-31-about/. Accessed June 2019.

31. McKenzie L, van der Pol M. Mapping the EORTC QLQ C-30 onto the EQ-5D instrument: the potential to estimate QALYs without generic preference data. Value Health. 2009;12(1):167-71. https ://doi.org/10.1111/j.1524-4733.2008.00405.x.

32. IBM. IBM Micromedex Red Book.

33. Janssen. Final 2018 Medicare coding and payment: drug administration services under the physician fee schedule; 2018. https:// www.janssencarepath.com/sites/www.janssencarepath.com/files /medicare-coding-and-payment-drug-admin-services-physicianfee-schedule.pdf. Accessed June 2019.

34. Optum. Soliris utilization: an EHR and claims analysis-prepared for Alexion Pharmaceuticals Inc. October 2018 (data on file)

35. Centers for Medicare and Medicaid Services. Final outpatient payment policies for 2018. https://www.aabb.org/advocacy/reimb ursementinitiatives/Documents/OPPS-2018-FINAL-rule-summa ry.pdf. Accessed June 2019.

36. Sanders GD, Neumann PJ, Basu A, Brock DW, Feeny D, Krahn $\mathrm{M}$, et al. Recommendations for conduct, methodological practices, and reporting of cost-effectiveness analyses: second panel on costeffectiveness in health and medicine. JAMA. 2016;316(10):1093103. https://doi.org/10.1001/jama.2016.12195.
37. Jang JH, Kim JS, Yoon SS, Lee JH, Kim YK, Jo DY, et al. Predictive factors of mortality in population of patients with paroxysmal nocturnal hemoglobinuria $(\mathrm{PNH})$ : results from a Korean PNH registry. J Korean Med Sci. 2016;31(2):214-21. https://doi. org/10.3346/jkms.2016.31.2.214.

38. Alexion Pharmaceuticals Inc. Haematology reimbursement advisory board. Leeds; 2018.

39. Loschi M, Porcher R, Barraco F, Terriou L, Mohty M, de Guibert $\mathrm{S}$, et al. Impact of eculizumab treatment on paroxysmal nocturnal hemoglobinuria: a treatment versus no-treatment study. Am J Hematol. 2016;91(4):366-70. https://doi.org/10.1002/ajh.24278.

40. Hearmon NC, Ghosh W, Buguth B, Kusel J. Model types submitted to NICE: what is considered appropriate by evidence review groups? Value Health. 2017;20(9):A752.

41. Roberts M, Russell LB, Paltiel AD, Chambers M, McEwan P, Krahn M, et al. Conceptualizing a model: a report of the ISPORSMDM Modeling Good Research Practices Task Force-2. Med Decis Making. 2012;32(5):678-89. https://doi.org/10.1177/02729 89X12454941.

42. ISPOR Health Economic Evaluations Publication Guidelines Good Reporting Practices Task Force. CHEERS checklist. https ://www.equator-network.org/wp-content/uploads/2013/04/Revis ed-CHEERS-Checklist-Oct13.pdf. Accessed Apr 2020.

43. Longworth L, Yang Y, Young T, Mulhern B, Hernandez Alava M, Mukuria $\mathrm{C}$, et al. Use of generic and condition-specific measures of health-related quality of life in NICE decision-making: a systematic review, statistical modelling and survey. Health Technol Assess. 2014;18(9):1-224. https://doi.org/10.3310/hta18090.

44. Socie G, Schrezenmeier H, Muus P, Lisukov I, Roth A, Kulasekararaj $\mathrm{A}$, et al. Changing prognosis in paroxysmal nocturnal haemoglobinuria disease subcategories: an analysis of the International PNH Registry. Intern Med J. 2016;46(9):1044-53. https://doi. org/10.1111/imj.13160. 